\title{
Efficient Adaptive Algorithms for DOA Estimation in Wireless Communications
}

\author{
J. G. ARCEO-OLAGUE ${ }^{1}$, D. H. COVARRUBIAS-ROSALES ${ }^{2}$, J. M. LUNA-RIVERA ${ }^{3}$, \\ A. ÁNGELES-VALENCIA ${ }^{4}$ \\ ${ }^{1}$ Electrical Engineering, Communications and Electronics, UAZ, Zacatecas, México \\ ${ }^{2}$ Electronics and Telecommunication Department, CICESE Research Center, Ensenada, México \\ ${ }^{3}$ Electronics Department, College of Sciences, UASLP, San Luis Potosi, México \\ ${ }^{4}$ CITEDI Research Center, Tijuana, México \\ Email:arceojg@ymail.com,dacoro@cicese.mx,mlr@fciencias.uaslp.mx,alfang@ieee.org \\ Received October 29, 2009; revised December 21, 2009; accepted December 28, 2009
}

\begin{abstract}
The problem of direction-of-arrival (DOA) estimation in mobile communication systems requires efficient algorithms with a high spatial resolution and a low computational complexity when moving sources are considered. MUSIC is known as a high resolution algorithm for DOA estimation but, this method demands high computational complexity to compute the signal subspace of the time-varying data of the correlation matrix. This paper focuses on MUSIC using subspace tracking methods, such as Bi-SVD and PAST, to carry out iterative DOA estimation. Accuracy and the processing time of both methods are evaluated and compared with the results of MUSIC. These results show the potential of Bi-SVD and PAST to reduce the processing time and to improve the accuracy when the number of snapshots and the source angular variation increases, assessing the source location for a dynamic mobile cellular environment.
\end{abstract}

Keywords: Direction-of-Arrival, Accuracy, Computational Complexity, Subspace Tracking, Array Antennas

\section{Introduction}

In mobile communication systems source localization with distributed sensor arrays can be performed by estimating the direction of arrival (DOA) of the signals coming from mobile terminals (sources). DOA is one of the most challenging problems which one has to solve for localizing and tracking multiple moving source as in radar, mobile communications and in other areas.

Within the class of high-resolution source localization methods, the MUSIC (MUltiple SIgnal Classification) method [1] has received the most attention and has been widely studied [2-4]. However, when the problem of moving sources is addressed, this method demands heavy computational load due to the decomposition of the subspace of the vectors of the correlation matrix, which is estimated based on an N-size sample [4-7]. For fast DOA tracking, the subspace tracking methods iteratively estimate the signal subspace which in this case is involved in the MUSIC spectrum. To address this problem, Bi-SVD (Bi-Iteration Singular-Value Decomposition) $[4,5]$ and PAST (Projection Approximation Subspace Tracking) [6] have been proposed and investigated due to their capabilities of successively updating (tracking) eigenvectors in the signal subspace of a correlation matrix [7-9]. Compared with the rectangular window in MUSIC conventional, in this signal subspace algorithms an exponential window is used for the signal processing. This window considers a forgetting factor to reduce the effect of the past observations which are down-weighted.

In this work, we present and evaluate a scheme that incorporates those subspace tracking methods into MUSIC, and shows a comparison of DOA successive estimation performance. For a small sample size in [4] is found that all the methods show the same root mean square error (RMSE) after convergence, and that all the methods can satisfactorily estimate DOAs. In contrast, we use a larger sample size to estimate the DOA considering the source's mobility. Compared with MUSIC conventional, our experimental analysis on simulated data demonstrates the accuracy improvement of both Bi-SVD and PAST algorithms to assess the sources DOA for a dynamic environment in terms of the root mean square error (RMSE).

\section{System Model}

Let $\boldsymbol{x}(t)$ be the $K x l$ data vector at the output of an uni- 
form linear array of $K$ elements spaced a distance $d$ between two consecutive elements. Assuming that the array of $K$ sensors receive $D$ narrowband signal waves from far-field sources with the same known center frequency, then the output vector $\boldsymbol{x}(t)$ can be described as:

$$
\boldsymbol{x}(t)=\sum_{l=0}^{D-1} s_{l}(t) \boldsymbol{a}_{l}\left(\theta_{l}\right)+\boldsymbol{n}(t)=\boldsymbol{A s}(t)+\boldsymbol{n}(t)
$$

where the $D x 1$ vector $s(t)=\left[s_{0}(t), \ldots, s_{D-1}(t)\right]^{\mathrm{T}}$ and the $K x 1$ vector $\boldsymbol{n}(t)=\left[n_{1}(t), \ldots, n_{K}(t)\right]^{\mathrm{T}}$ denote the complex amplitudes of the signals and the measurement noise at time $t$. The $K x D$ steering matrix $\boldsymbol{A}$ is composed by $D$ steering vectors $(K x l)$, with $\boldsymbol{a}_{l}\left(\theta_{l}\right)=\left[1, \exp \left(-j(2 \pi / \lambda) d \operatorname{sen} \theta_{l}\right), \ldots\right.$, $\left.\exp \left(-j(2 \pi / \lambda) d(K-1) \operatorname{sen} \theta_{l}\right)\right], \theta_{l}$ is the direction of arrival and $\lambda$ is the carrier wavelength. The noise samples are assumed to be zero mean with variance $\sigma^{2}$.

From the eigen decomposition of the correlation matrix $\boldsymbol{R}=E\left[\boldsymbol{x}(t) \boldsymbol{x}^{H}(t)\right]$ are obtained $\boldsymbol{E}_{s}(t)$ and $\boldsymbol{E}_{n}(t)$ corresponding to the signal and noise subspaces respectively, [1]. The signal subspace is formed by the $D$ eigenvectors corresponding to the $D$ largest eigenvalues. Usually, the MUSIC spectrum is computed with the noise subspace to improve the spectral resolution. Besides that, the subspace tracking algorithms approaches the signal subspace $\boldsymbol{E}_{s}(t)$, then, the identity $\boldsymbol{E}_{n}(t) \boldsymbol{E}_{n}(t)^{H}=\boldsymbol{I}-\boldsymbol{E}_{s}(t) \boldsymbol{E}_{s}(t)^{H}$ must be employed to track the DOA from the MUSIC spectrum as follows [4]:

$$
P_{M U}(\theta, t)=\frac{1}{\boldsymbol{a}^{H}(\theta)\left(\boldsymbol{I}-\boldsymbol{E}_{s}(t) \boldsymbol{E}_{s}(t)^{H}\right) \boldsymbol{a}(\theta)}
$$

In what follows, are explained Bi-SVD and PAST algorithms for the $\boldsymbol{E}_{s}(t)$ signal subspace tracking.

\subsection{Bi-SVD Algorithm}

Let $\boldsymbol{x}(t)$ be a random complex process with the correlation matrix $\boldsymbol{R}=\mathrm{E}\left[\boldsymbol{x}(t) \boldsymbol{x}(t)^{\mathrm{H}}\right]$ and the signal subspace $\boldsymbol{E}_{\mathrm{s}}(t)$ contains the $D$ dominant eigenvectors. For subspace tracking, the data matrix $\boldsymbol{X}(t)$ can be updated in the time according [5]:

$$
\boldsymbol{X}(t)=\left[\begin{array}{c}
(1-\beta)^{1 / 2} \boldsymbol{x}^{T}(t) \\
\beta^{1 / 2} \boldsymbol{X}(t-1)
\end{array}\right]
$$

where $\boldsymbol{x}(t)$ is the current data vector, $0<\beta<1$ is the forgetting factor and $X(t-1)$ is the data matrix at time instant $t-1$. Consider the following bi-iteration applied on the (NxK) data matrix $\boldsymbol{X}(t)$ as given in [5]:

$$
\boldsymbol{Q}_{A}(0)=\left[\begin{array}{c}
\boldsymbol{I}_{D} \\
\boldsymbol{0}
\end{array}\right] ;\left[\begin{array}{c}
\boldsymbol{B}(t)=\boldsymbol{X}(t) \boldsymbol{Q}_{A}(t-1) \\
\frac{\boldsymbol{B}(t)=\boldsymbol{Q}_{B}(t) \boldsymbol{R}_{B}(t)}{\boldsymbol{A}(t)=\boldsymbol{X}^{H}(t) \boldsymbol{Q}_{B}(t)} \\
\boldsymbol{A}(t)=\boldsymbol{Q}_{A}(t) \boldsymbol{R}_{A}(t)
\end{array}\right],
$$

where $\boldsymbol{Q}_{\mathrm{A}}(0)$ is the initial value for $\boldsymbol{Q}_{\mathrm{A}}(\mathrm{t})$. Here, $\boldsymbol{Q}_{\mathrm{A}}(\mathrm{t})$ denotes an estimate of the signal subspace $\boldsymbol{E}_{\mathrm{s}}(t), \boldsymbol{R}_{\mathrm{A}}(\mathrm{t})$ and $\boldsymbol{R}_{\mathrm{B}}(\mathrm{t})$ are $D$-dimensional upper-triangular matrices obtained from QR decomposition. Notice that the data matrix $\boldsymbol{X}(t)$ in (3) is updated recursively then it results in a growing matrix. A solution of this problem is to approximate $\boldsymbol{X}(t)$ as [5]:

$$
\hat{\boldsymbol{X}}(t)=\boldsymbol{Q}_{B}(t) \boldsymbol{R}_{B}(t) \boldsymbol{Q}_{A}^{H}(t-1) .
$$

With the suboptimal approximation in (4), a fast exponential window based Bi-SVD subspace tracking algorithm was developed in [5]. Now, projecting $\boldsymbol{x}(t)$ into the space spanned by $\boldsymbol{Q}_{\mathrm{A}}(\mathrm{t}-1)$ the complement of its orthogonal projection is

$$
\boldsymbol{x}_{\perp}(t)=\boldsymbol{x}(t)-\boldsymbol{Q}_{A}(t-1) \boldsymbol{h}(t)
$$

where $\boldsymbol{h}(t)=\boldsymbol{Q}_{A}^{H}(t-1) \boldsymbol{x}(t)$. The normalization of $\boldsymbol{x}_{\perp}(\mathrm{t})$ results in

$$
\overline{\boldsymbol{x}}_{\perp}(t)=e_{x}^{-1 / 2}(t) \boldsymbol{x}_{\perp}(t),
$$

with $e_{x}(t)=\boldsymbol{x}_{\perp}^{H}(t) \boldsymbol{x}_{\perp}(t)$. From (5), the decomposition for $\boldsymbol{x}(t)$ becomes:

$$
\boldsymbol{x}(t)=e_{x}^{1 / 2}(t) \overline{\boldsymbol{x}}_{\perp}(t)+\boldsymbol{Q}_{A}(t-1) \boldsymbol{h}(t) .
$$

Using (5) and (8) into (3) and then substituting $\boldsymbol{X}(t)$ in (4), we obtain $\boldsymbol{Q}_{\mathrm{A}}(\mathrm{t})$ after several computations as follows:

$$
\boldsymbol{Q}_{A}(t)=\boldsymbol{Q}_{A}(t-1) \boldsymbol{\Theta}_{A}(t)+\overline{\boldsymbol{x}}_{\perp}(t) \boldsymbol{f}^{H}(t),
$$

where

$$
\begin{gathered}
\boldsymbol{f}(t)=\boldsymbol{Q}_{A}^{H}(t) \overline{\boldsymbol{x}}_{\perp}(t) \\
\boldsymbol{\Theta}_{A}(t)=\boldsymbol{Q}_{A}^{H}(t-1) \boldsymbol{Q}_{A}(t)
\end{gathered}
$$

In Equation 9, the subspaces $\boldsymbol{R}_{\mathrm{A}}(t), \boldsymbol{R}_{\mathrm{B}}(t)$ and $\boldsymbol{\Theta}_{\mathrm{A}}(t)$ are updated successively with initial values $\boldsymbol{R}_{\mathrm{A}}(0)=\mathbf{0}$, $\boldsymbol{R}_{\mathrm{B}}(0)=\boldsymbol{I}_{\mathrm{D}}$ and $\boldsymbol{\Theta}_{\mathrm{A}}(0)=\boldsymbol{I}_{\mathrm{D}}$.

\subsection{PAST Algorithm}

The PAST algorithm is based on the idea that $\boldsymbol{Q}_{\mathrm{A}}(\mathrm{t})$ is the signal subspace when is minimized (12).

$$
J\left(\boldsymbol{Q}_{A}(t)\right)=\sum_{i=1}^{t} \beta^{t-i}\left\|\boldsymbol{x}(i)-\boldsymbol{Q}_{A}(t) \boldsymbol{Q}_{A}{ }^{H}(t) \boldsymbol{x}(i)\right\|^{2}
$$

An exponentially weighted sum is considered into $J\left(\boldsymbol{Q}_{\mathrm{A}}(t)\right)$, where $\beta$ is the forgetting factor. At time $t$, all sample vectors are involved in the estimation of the signal subspace. The algorithm PAST approximates $\boldsymbol{Q}_{\mathrm{A}}{ }^{\mathrm{H}}(t) \boldsymbol{x}(i)$ in (12) with $\boldsymbol{y}(\mathrm{i})=\boldsymbol{Q}_{\mathrm{A}}{ }^{\mathrm{H}}(i-1) \boldsymbol{x}(\mathrm{i})$ by using the unknown projection of $\boldsymbol{x}(i)$ into the columns of $\boldsymbol{Q}_{\mathrm{A}}(t)$ [6]. The approximated cost function is then given by:

$$
J^{\prime}\left(\boldsymbol{Q}_{A}(t)\right)=\sum_{i=1}^{t} \beta^{t-i}\left\|\boldsymbol{x}(i)-\boldsymbol{Q}_{A}(t) \boldsymbol{y}(i)\right\|^{2}
$$


The choice of $\boldsymbol{Q}_{A}(\mathrm{t})$ that minimize $J^{\prime}\left(\boldsymbol{Q}_{A}(\mathrm{t})\right)$ is [6]

$$
\begin{aligned}
\boldsymbol{Q}_{A}(t) & =\boldsymbol{C}_{x y}(t) \boldsymbol{C}_{y y}^{-1}(t) \\
\boldsymbol{C}_{x y}(t) & =\beta \boldsymbol{C}_{x y}(t-1)+\boldsymbol{x}(t) \boldsymbol{y}^{H}(t) \\
\boldsymbol{C}_{y y}(t) & =\beta \boldsymbol{C}_{y y}(t-1)+\boldsymbol{y}(t) \boldsymbol{y}^{H}(t)
\end{aligned}
$$

Therefore, the minimization process yields that $J\left(\boldsymbol{Q}_{\mathrm{A}}(t)\right)$ on only internal noise, resulting in $\boldsymbol{Q}_{\mathrm{A}}(t)$ equal to the signal subspace matrix $\boldsymbol{E}_{\mathrm{s}}(t)$, obtained recursively from (14).

\section{Simulation Results}

Successive update algorithms using Bi-SVD and PAST into MUSIC are compared in estimation accuracy with the conventional MUSIC. The estimation accuracy is evaluated by RMSE (Root Mean Square Error), defined as follows:

$$
\operatorname{RMSE}(t)=\sqrt{\frac{1}{D} \sum_{l=1}^{D}\left\{\frac{1}{P} \sum_{p=1}^{P}\left(\hat{\theta}_{l p}(t)-\theta_{l}\right)^{2}\right\}}
$$

where $\hat{\theta}_{l p}$ defines the estimated DOAs, D denotes the

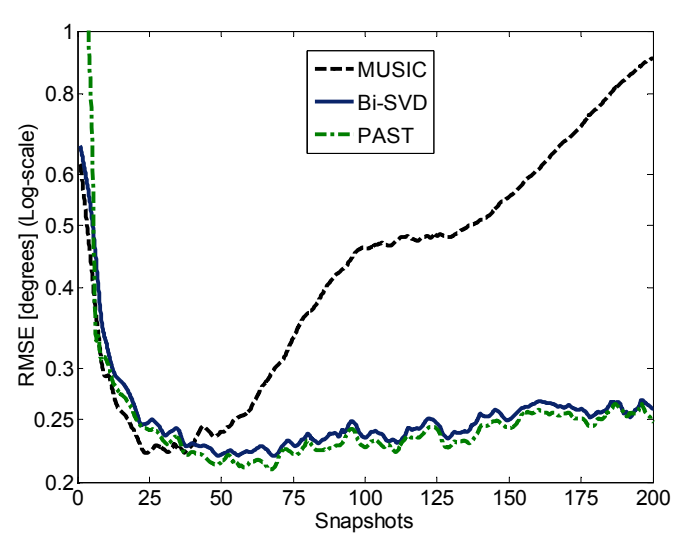

Figure 1. RMSE evaluation in DOA estimation (dynamic environment). $\mathrm{SNR}=20 \mathrm{~dB}$ spatial variation $\delta=0.01^{\circ}$.

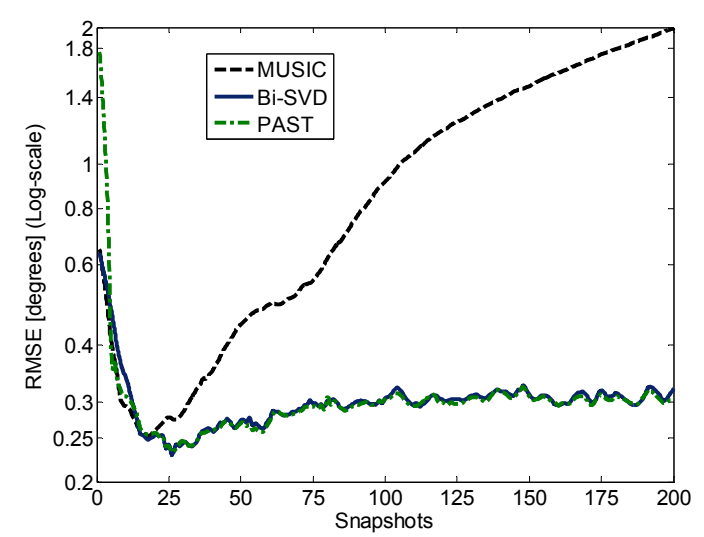

Figure 2. RMSE evaluation in DOA estimation (dynamic environment). $\mathrm{SNR}=20 \mathrm{~dB}$ spatial variation $\delta=0.02^{\circ}$.

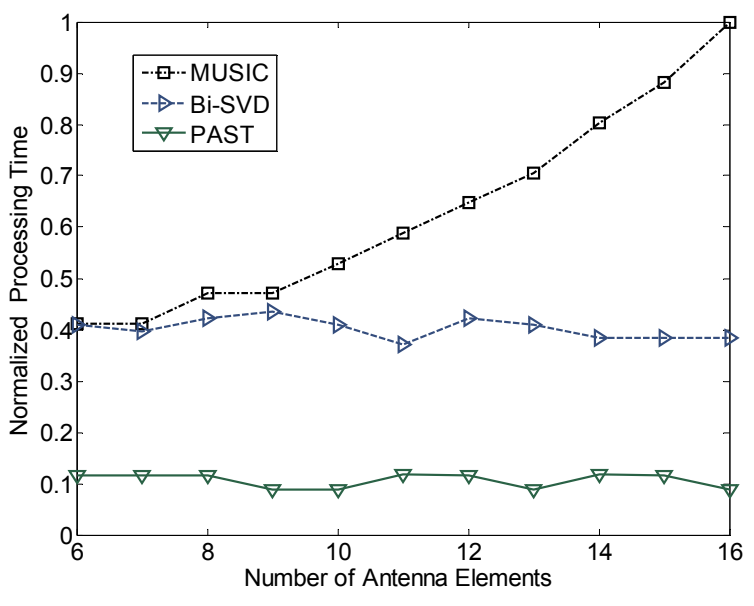

Figure 3. Normalized processing time. Signal subspace estimation considering variation of the number of array antenna elements.

number of sources and $\mathrm{P}$ the number of independent trials.

For the simulations, we consider a uniform linear array (ULA) of $\mathrm{K}=10$ sensors separated a half wavelength of the actual source signals. We assume the case of two narrowband signals in the far-field, located at $\left[-30^{\circ}, 50^{\circ}\right]$ and separated from the antenna array by $930 \mathrm{~m}$ and $425 \mathrm{~m}$ respectively, transmitting with the same power and the same carrier frequency of $f=1900 \mathrm{MHz}$. For this case, the number of independent trials is set to $P=1000$, assuming a forgetting factor of $\beta=0.9$.

Simulation results are obtained by incorporating over time the sources' spatial angular variation $\delta$ as the dynamic component. Figure 1 compares the RMSE obtained by the PAST, Bi-SVD and MUSIC methods in a dynamic environment. For each snapshot, the angular variation $\delta$ is increased a factor of 0.01 degrees, that is, the sources location at time $t$ are given by $\left[-30^{\circ}+t \delta, 50^{\circ}+\right.$ $t \delta$. The results in Figure 1 show a small RMSE for PAST and Bi-SVD methods. After a 40 snapshots the accuracy of the MUSIC method is degraded with an accumulated RMSE value of $0.9^{\circ}$ approximately. On the other hand, for Bi-SVD and PAST, their RMSE error increases slowly until a value close to $0.26^{\circ}$. In this case, comparing the subspace tracking algorithms into MUSIC with the conventional, they improve the accuracy as the number of snapshots increases.

In a second case, is considered an increase of angular variation $\left(\delta=0.02^{\circ}\right)$. From Figure 2, we found that after 20 snapshots MUSIC conventional rapidly increases its RMSE error from $0.25^{\circ}$ to $2^{\circ}$ on the maximum number of snapshots considered. In this case, the accuracy of the MUSIC method is remarkably worse compared with the maximum RMSE error of $0.3^{\circ}$ obtained by the subspace tracking algorithms. The forgetting factor used by subspace tracking algorithms improved the accuracy 
into MUSIC to estimate the DOA, while MUSIC conventional fails as the number of snapshots and angular variation increases.

Finally, the processing time of the methods is evaluated and normalized taking into account the maximum processing time. In Figure 3 the processing time is normalized with the maximum time obtained during the simulations. We observe that the processing time of the PAST algorithm is smaller than both Bi-SVD and MUSIC. More interestingly, the results show that PAST and Bi-SVD processing times are independent of the number of antenna array elements, situation that does not hold by MUSIC which grows linearly with the number of antenna elements.

\section{Conclusions}

The PAST and Bi-SVD subspace-tracking algorithms were applied to MUSIC in order to face the accuracy and processing time problems in DOA estimation. The simulations show good results as far as the application of the subspace-tracking algorithms. Our experimental analysis on simulated data demonstrates the potential reduction in processing time and accuracy improvement to assess the sources DOA when the number of snapshots and angular variation increases. It is shown that as source mobility increases, it produces a higher error for MUSIC conventional. However, the PAST and Bi-SVD algorithms show a lower estimation error, varying quietly with the mobility of sources. On the other hand, the processing time of PAST algorithm is smaller than both Bi-SVD and MUSIC conventional. The processing time required by these subspace tracking algorithms is independent of the number of antenna array elements.

\section{Acknowledgments}

This work was supported by PROMEP and the Mexican National Science and Technology Council, CONACyT, under Grant 52374.

\section{References}

[1] R. O. Schmidt, "Multiple emitter location and signal parameter estimation," IEEE Transactions Antennas and Propagation, Vol. 34, pp. 276-280, 1986

[2] D. H. Covarrubias and J. G. Arceo, "Improving resolution on DOA estimation in a multipath macrocell environment," Proc. IEEE SympoTIC, Bratislava Slovak Republic, pp. 39-42, 2004.

[3] J. G. Arceo, D. H. Covarrubias, and J. M. Luna, "Efficiency evaluation of the unconditional maximum likelihood estimator for near-field source DOA estimation," ETRI Journal, Vol. 28, pp. 761-769, 2006.

[4] N. Kikuma, "Iterative DOA estimation using subspace tracking methods and adaptative beamforming," IEICE Transactions on Communication, Vol. E88-B, pp. 1818 1828,2005

[5] P. Strobach, "Bi-Iteration SVD subspace tracking algorithms," IEEE Trans. Signal Processing, Vol. 45, pp. 1222 1240, 1997.

[6] B. Yang, "Projection approximation subspace tracking," IEEE Transactions Signal Proceedings, Vol. 43, pp. 95107, 1995.

[7] P. Strobach, "Bi-Iteration multiple invariance subspace tracking and adaptive ESPRIT," IEEE Transactions on Signal Processing, Vol. 48, pp. 442-456, 2000.

[8] A. Kuchar, M. Tangemann, and E. Bonek, "A real-time DOA-based smart antenna processor," IEEE Transactions on Vehicular Technology, Vol. 51, pp. 1279-1293, 2002.

[9] S. Ouyang and Y. Hua, "Bi-Iterative least-square method for subspace tracking," IEEE Transactions on Signal Processing, Vol. 53, pp. 2984-2996, 2005. 